\title{
STRICT QUANTIZATIONS OF ALMOST POISSON MANIFOLDS
}

\author{
HANFENG LI
}

\begin{abstract}
We show the existence of (non-Hermitian) strict quantization for every almost Poisson manifold.
\end{abstract}

\section{INTRODUCTION}

In the passage from classical mechanics to quantum mechanics, smooth functions on symplectic manifolds (more generally, Poisson manifolds) are replaced by operators on Hilbert spaces, and the Poisson bracket of smooth functions are replaced by commutators of operators. When one thinks of classical mechanics as limits of quantum mechanics, the Poisson bracket becomes limits of commutators. Based on the general theory of formal deformations of algebras 9], F. Bayer et al. [1] initiated the study of deformation quantization of Poisson manifolds.

Let $M$ be a Poisson manifold. Denote $C^{\infty}(M)$ the space of smooth $\mathbb{C}$-valued functions on $M$, and denote $C^{\infty}(M)[[\hbar]]$ the space of formal power series with coefficients in $C^{\infty}(M)$. Recall that a star product on $M$ is a $\mathbb{C}[[\hbar]]$-bilinear associative multiplication $*$ on $C^{\infty}(M)[[\hbar]]$ of the form

$$
f * g=\sum_{r=0}^{\infty} C_{r}(f, g) \hbar^{r}, \text { for } f, g \in C^{\infty}(M),
$$

where $C_{0}(f, g)=f g, f * g-g * f \equiv\{f, g\} i \hbar \bmod \hbar^{2}$, and each $C_{r}(\cdot, \cdot)$ is a bidifferential operator. The algebra $\left(C^{\infty}(M)[[\hbar]], *\right)$ is called a deformation quantization of $M$. The existence of deformation quantizations for any symplectic manifold was proven first by De Wilde and Lecomte [7. The general case of Poisson manifolds was proven by Kontsevich 12.

In deformation quantizations $\hbar$ is only a formal parameter, and elements in $C^{\infty}(M)[[\hbar]]$ are not operators on Hilbert spaces. In order to study quantizations in a stricter sense, Rieffel introduced strict deformation quantization of Poisson manifolds [23, 24], and showed that noncommutative tori arise naturally as strict deformation quantizations of certain Poisson brackets on the ordinary torus. Later, Landsman introduced a weaker notion strict quantization to accommodate some other interesting examples such as Berezin-Toeplitz quantization of Kähler manifolds. Recall the definition of strict quantization as formulated in [27] 13]:

Definition 1.1. Let $M$ be a Poisson manifold, and let $C_{\infty}(M)$ be the algebra of continuous functions on $M$ vanishing at $\infty$. By a strict quantization of $\mathrm{M}$ we mean a dense $*$-subalgebra $A$ of $C_{\infty}(M)$ closed under the Poisson bracket, together with a continuous field of $C^{*}$-algebras $\mathcal{A}_{\hbar}$ over a closed subset $I$ of the real line containing 0 as a non-isolated point, and linear maps $\pi_{\hbar}: A \rightarrow \mathcal{A}_{\hbar}$ for each $\hbar \in I$, such that

(1) $\mathcal{A}_{0}=C_{\infty}(M)$ and $\pi_{0}$ is the canonical inclusion of $A$ into $C_{\infty}(M)$,

Date: May 18, 2003. 
(2) the section $\left(\pi_{\hbar}(f)\right)$ is continuous for every $f \in A$,

(3) for all $f, g \in A$ we have

$$
\lim _{\hbar \rightarrow 0}\left\|\left[\pi_{\hbar}(f), \pi_{\hbar}(g)\right] /(i \hbar)-\pi_{\hbar}(\{f, g\})\right\|=0 .
$$

If each $\pi_{\hbar}$ is injective, we say that the strict quantization is faithful. If $A \supseteq$ $C_{c}^{\infty}(M)$, the space of compactly supported smooth functions on $M$, we also say that the strict quantization is flabby. If $\left(\pi_{\hbar}(f)\right)^{*}=\pi_{\hbar}\left(f^{*}\right)$ for all $\hbar \in I$ and $f \in A$, we say that the strict quantization is Hermitian. When a Lie group has an smooth action $\alpha$ on $M$ preserving the Poisson bracket, if $G$ also has an continuous action $\beta_{\hbar}$ on each $\mathcal{A}_{\hbar}$ such that $\beta_{0}=\alpha^{*}$ and the maps $\pi_{\hbar}$ are all $G$-equivariant, we say that the strict quantization is $G$-equivariant. When the strict quantization is faithful and $\pi_{\hbar}(A)$ is a $*$-subalgebra of $\mathcal{A}_{\hbar}$ for each $\hbar$, it's called a strict deformation quantization.

Strict quantizations have been constructed for several classes of Poisson manifolds such as Poisson manifolds coming from actions of $\mathbb{R}^{d}[26$, quantizable compact Kähler manifolds [2], dual of integrable Lie algebroids [25, 15], compact Riemannian surfaces of genus $\geq 2$ [11, 17, 18, etc. These constructions are all global, and the resulting strict quantizations are Hermitian. However, the progress of the study of strict quantizations is much slower compared with that of deformation quantizations-so far there is even no existence result for general symplectic manifolds. Recently Natsume et al. [19] constructed strict quantizations for every compact symplectic manifold $M$ satisfying the topological conditions that $\pi_{1}(M)$ is exact and $\pi_{2}(M)=0$. Roughly speaking, they use partition of unity to reduce $M$ to Darboux charts, where they can use the Moyal-Weyl product. Thus their construction is local. It turns out that the resulting strict quantizations are not Hermitian.

Recall that an almost Poisson manifold is a smooth manifold $M$ equipped with some $\Pi \in \Gamma\left(\wedge^{2} T M\right)[\underline{6}$. In this case, we can still define a bracket $\{f, g\}=\Pi(d f, d g)$ for $f, g \in C^{\infty}(M)$, which is bilinear and skew-symmetric, and satisfies the Leibniz rule. And the bracket satisfies the Jacobi identity if and only if $M$ equipped with this bracket is actually a Poisson manifold. Clearly we can also talk about strict quantizations of almost Poisson manifolds. The main result of this paper is the following:

Theorem 1.2. Let $(M, \Pi)$ be an almost Poisson manifold, and let $\alpha$ be a smooth action of a Lie group $G$ on $M$ preserving the bracket. If $M$ has a $G$-invariant Riemannian metric, then $M$ has a $G$-equivariant faithful flabby strict quantization over $I=[0,1]$ with $A=C_{c}^{\infty}(M)$. In particular, taking $G=\{e\}$ we see that $M$ has a faithful flabby strict quantization over $[0,1]$ with $A=C_{c}^{\infty}(M)$.

Our construction is also local, but different from the one in [19. Actually we shall construct a locally trivial $C^{*}$-algebra bundle over $M$ in a canonical way, thus don't need local charts and partition of unity. But our strict quantizations are not Hermitian either.

This paper is organized as follows. Though our construction for strict quantizations of almost Poisson manifolds is only slightly more complicated for that of symplectic manifolds, the idea is most natural in the case of symplectic manifolds. So we prove Theorem 1.2 for symplectic manifolds first in Section 2 Then we prove Theorem 1.2 for the general case in Section 3 Our construction depends on the 
choice of an inner product on the vector bundle $T^{*} M \oplus T M$. We define homotopy of strict quantizations in Section 4 and show that the homotopy class of our strict quantizations doesn't depend on the choice of the inner products. In Section 5 we define local strict quantizations, and show that they can't be Hermitian. We also show that our strict quantizations can't be restricted to a $*$-subalgebra of $A$ to get a strict deformation quantization of $M$ unless $\Pi=0$. This gives a negative answer to a question of Rieffel [27, Question 25]. In Section 6 we discuss certain functorial properties of our construction. All of our construction is based on the existence of asymptotic representations of Heisenberg commutation relations (Definition 2.1). We prove the existence of such asymptotic representations in Section 7

Acknowledgments. I would like to thank Marc Rieffel for many helpful discussions and suggestions. I also thank Henrique Bursztyn for valuable discussions about deformation quantizations.

\section{Strict Quantizations of Symplectic Manifolds}

Throughout this paper, for a continuous field $D$ of $C^{*}$-algebras $\left\{D_{\hbar, x}\right\}_{x \in X}$ over a locally compact Hausdorff space $X$ we denote $\Gamma_{\infty}(D)$ the algebra of continuous sections of $D$ vanishing at $\infty$ [ .

We show the main idea of our construction first. Let $(M, \omega)$ be a symplectic manifold, and let $\Pi \in \Gamma\left(\wedge^{2} T M\right)$ be the corresponding bivector field as usual. Let $f, g \in C_{c}^{\infty}(M)$. Since we think of $\mathcal{A}_{\hbar}$ as deformations of $C_{\infty}(M)$, we would like to write $\pi_{\hbar}(f)$ as $f+\tau_{\hbar}(f)$, which makes sense when $\mathcal{A}_{\hbar}$ contains $C_{\infty}(M)$ as a $C^{*}$-subalgebra, and assume that

$$
\left\|\tau_{\hbar}(f)\right\| \rightarrow 0
$$

as $\hbar \rightarrow 0$. Assume further that $C_{\infty}(M)$ lies in the center of $\mathcal{A}_{\hbar}$. Then

$$
\left[\pi_{\hbar}(f), \pi_{\hbar}(g)\right] /(i \hbar)-\pi_{\hbar}(\{f, g\})=\left[\tau_{\hbar}(f), \tau_{\hbar}(g)\right] /(i \hbar)-\{f, g\}-\tau_{\hbar}(\{f, g\}) .
$$

Thus the condition (3) in Definition 1.1 becomes $\left\|\left[\tau_{\hbar}(f), \tau_{\hbar}(g)\right] /(i \hbar)-\{f, g\}\right\| \rightarrow 0$. Notice that $\{f, g\}$ doesn't depend on $f$ and $g$, but depends only on $d f$ and $d g$. So we would like to assume that $\tau_{\hbar}(f)$ depends only on $d f$ linearly. Then we attempt to write $\tau_{\hbar}(f)$ as $\hbar^{\frac{1}{2}} \varphi_{\hbar}(d f)$, where $\varphi_{\hbar}: \Gamma\left(T^{*} M\right) \rightarrow \mathcal{A}_{\hbar}$ is a linear map. Now $\left\|\left[\tau_{\hbar}(f), \tau_{\hbar}(g)\right] /(i \hbar)-\{f, g\}\right\| \rightarrow 0$ becomes

$$
\left\|\left[\varphi_{\hbar}(d f), \varphi_{\hbar}(d g)\right]-\Pi(d f, d g) i\right\| \rightarrow 0 .
$$

We assume further that $\mathcal{A}_{\hbar}=\Gamma_{\infty}(D)$ for some continuous field of $C^{*}$-algebras $\left\{D_{\hbar, x}\right\}_{x \in M}$ over $M$, and that $D_{\hbar, x}$ contains $T^{*} M_{x}$ as a linear subspace with $\varphi_{\hbar}$ being just pointwise embedding. Then (2) becomes

$$
\left\|[u, v]-\Pi_{x}(u, v) i\right\|_{D_{\hbar, x}} \rightarrow 0 .
$$

for all $u, v \in T^{*} M_{x}$.

This leads to our definition of asymptotic representation of Heisenberg commutation relations and that of Heisenberg $C^{*}$-algebra $\mathfrak{A}_{2 n}$ (which will be $\mathcal{A}_{\hbar, x}$ for $2 n=\operatorname{dim} M)$ in Definition 2.1 below. In order to embed $T^{*} M_{x}$ into $\mathfrak{A}_{2 n}$ without referring to local basis, we also need an action of the structure group of $T^{*} M$ on $\mathfrak{A}_{2 n}$. If we consider only $\Pi$, then the structure group is the symplectic linear group $S p(2 n)[5$, which is too big. By adding a compatible almost complex structure on $T^{*} M$ we can reduce the structure group to the unitary group $U(n)$ (see Lemma 2.5). Here we recall how $U(n)$ acts on $T^{*} M_{x}$. 
Let $V$ be a finite dimensional vector space over $\mathbb{R}$ with a symplectic structure $\omega$ and a compatible almost complex structure $J$ [5], i.e. $J: V \rightarrow V$ is linear satisfying that $J^{2}=-1$ and $\langle u, v\rangle:=\omega(u, J v)$ is an inner product on $V$. Say $\operatorname{dim} V=2 n$. Then we can always find basis $u_{1}, \cdots, u_{n}, v_{1}, \cdots, v_{n}$ of $V$ such that under this basis $\omega$ and $J$ have matrix forms $\left(\begin{array}{cc}0 & I \\ -I & 0\end{array}\right)$ and $\left(\begin{array}{rr}0 & -I \\ I & 0\end{array}\right)$ respectively. We'll call such basis unitary basis of $V$. Notice that a unitary basis is an orthonormal basis under the induced inner product. If we make $V$ into a complex vector space by $J$ and identify matrix $X+i Y \in M_{n \times n}(\mathbb{C})$, where $X, Y \in$ $M_{n \times n}(\mathbb{R})$, with $\left(\begin{array}{rr}X & -Y \\ Y & X\end{array}\right) \in M_{2 n \times 2 n}(\mathbb{R})$, then $U(n)$ is exactly the group of linear transformations on $V$ taking unitary basis to unitary basis.

Definition 2.1. Let $n \in \mathbb{N}$, and let $\mathbb{R}^{2 n}$ be equipped with the standard symplectic vector space structure $\Omega$ and the standard compatible almost complex structure, i.e. for the standard basis $e_{1}, \cdots, e_{2 n}$ being a unitary basis. By an asymptotic representation of Heisenberg commutation relations, we mean a unital $C^{*}$-algebra $\mathfrak{A}_{2 n}$ with a continuous action $\rho$ of $U(n)$ and a $U(n)$-equivariant $\mathbb{R}$-linear map $\varphi_{\hbar}$ : $\mathbb{R}^{2 n} \rightarrow \mathfrak{A}_{2 n}$ for each $0<\hbar \leq 1$ such that

(1) for any $u, v \in \mathbb{R}^{2 n}$ we have $\left[\varphi_{\hbar}(u), \varphi_{\hbar}(v)\right] \rightarrow \Omega(u, v) i$ as $\hbar \rightarrow 0$;

(2) the map $(0,1] \rightarrow B\left(\mathbb{R}^{2 n}, \mathfrak{A}_{2 n}\right)$ given by $\hbar \mapsto \varphi_{\hbar}$ is continuous, where $B\left(\mathbb{R}^{2 n}, \mathfrak{A}_{2 n}\right)$ is the Banach space of linear maps $\mathbb{R}^{2 n} \rightarrow \mathfrak{A}_{2 n}$;

(3) $\hbar^{\frac{1}{2}}\left\|\varphi_{\hbar}\right\| \rightarrow 0$ as $\hbar \rightarrow 0$;

(4) $\mathfrak{A}_{2 n}$ is generated by $\cup_{0<\hbar \leq 1} \varphi_{\hbar}\left(\mathbb{R}^{2 n}\right)$.

The $C^{*}$-algebra $\mathfrak{A}_{2 n}$ will be called a Heisenberg $C^{*}$-algebra of dimension $2 n$.

Remark 2.2. The condition (3) is not crucial. Given $\mathfrak{A}_{2 n}$ and $\varphi_{\hbar}$ satisfying the other conditions we can always reparameterize $\varphi_{\hbar}$ 's to make them satisfy (3).

The main technical part of our construction is the following:

Theorem 2.3. For each $n \in \mathbb{N}$ there exists a Heisenberg $C^{*}$-algebra $\mathfrak{A}_{2 n}$.

Theorem 2.3 will be proved in Section 7 From now on we'll fix a Heisenberg $C^{*}$-algebra $\mathfrak{A}_{2 n}$ for each $n \in \mathbb{N}$ unless stated otherwise.

We'll construct a $C^{*}$-algebra bundle $D$ over $M$ with fibres all isomorphic to $\mathfrak{A}_{2 n}$ and a bundle map $T^{*} M \rightarrow D$. Actually we shall do this construction more generally for almost symplectic bundles, which will be useful in Section 3

Definition 2.4. Let $E \rightarrow M$ be a real vector bundle, and let $\Pi \in \Gamma\left(\wedge^{2} E^{*}\right)$. We call the pair $(E, \Pi)$ an almost Poisson bundle over $M$. If $\Pi$ is nondegenerate everywhere we call it an almost symplectic bundle over $M$. For two almost Poisson bundles $\left(E, \Pi_{E}\right)$ and $\left(F, \Pi_{F}\right)$ over $M$ we call a bundle map $\psi: E \rightarrow F$ an almost Poisson map if $\Pi_{F}(\psi(f), \psi(g))=\Pi_{E}(f, g)$ for all $f, g \in \Gamma(E)$.

As the case for symplectic manifolds [5], one sees easily that every almost symplectic bundle $(E, \Pi)$ has compatible almost complex structures.

Lemma 2.5. Let $(E, \Pi)$ be an almost symplectic bundle over $M$ with a compatible almost complex structure $J$. Say $\operatorname{dim} E=2 n$. Then for any $x \in M$ there exists a neighborhood $U$ of $x$ and sections $f_{1}, \cdots, f_{n}, g_{1}, \cdots, g_{n}$ of $E$ on $U$ such that they are a unitary basis at each point of $U$. 
Proof. It is easy to see that we can find sections $f_{1}, \cdots, f_{n}, g_{1}, \cdots, g_{n}$ near $x$ such that under this basis, $\Pi$ is of the matrix form $\left(\begin{array}{cc}0 & I \\ -I & 0\end{array}\right)$ at each point. Let $E^{+}=\operatorname{span}\left\{f_{1}, \cdots, f_{n}\right\}$. Then near $x$ this is a subbundle of $E$, and $E_{y}^{+}$is a Lagrangian subspace of $E_{y}$ at each point $y$. Let $f_{1}^{\prime}, \cdots, f_{n}^{\prime}$ be $n$ sections of $E^{+}$ near $x$ such that $f_{1}^{\prime}(y), \cdots, f_{n}^{\prime}(y)$ is an orthonormal basis at each point $y$. Then $f_{1}^{\prime}, \cdots, f_{n}^{\prime}, J f_{1}^{\prime}, \cdots, J f_{n}^{\prime}$ satisfy the requirement.

Lemma 2.5 shows that the structure group of $(E, \Omega, J)$ is $U(n)$. Let $U(E)$ be the set of unitary basis of $E$ at all points, then $U(E)$ is a principle $U(n)$-bundle on $M$. As usual, the action $\rho$ of $U(n)$ on $\mathfrak{A}_{2 n}$ induces a $C^{*}$-algebra bundle $D=$ $U(E) \times_{U(n)} \mathcal{A}_{2 n}$ over $M$, which is the quotient of $U(E) \times \mathcal{A}_{2 n}$ by the relation $(a, T) \sim\left(a g, g^{-1} T\right)$ with $g \in U(n)$. Then $D$ has all fibres isomorphic to $\mathfrak{A}_{2 n}$. Notice that the induced vector bundle $E^{\prime}:=U(E) \times_{U(n)} \mathbb{R}^{2 n}$ has an induced almost symplectic structure and an induced almost complex structure. Clearly $E^{\prime}$ equipped with these structures is isomorphic to $(E, \Omega, J)$. For each $\hbar \in(0,1]$ the $U(n)$ equivariant linear map $\varphi_{\hbar}: \mathbb{R}^{2 n} \rightarrow \mathfrak{A}_{2 n}$ also induces a bundle map $E \cong E^{\prime} \rightarrow D$, which we still denote by $\varphi_{\hbar}$.

Definition 2.6. Let $(E, \Pi, J)$ be as in Lemma 2.5 We call the $C^{*}$-algebra bundle $D$ constructed above the quantization bundle of $(E, \Omega, J)$, and call the bundle maps $\varphi_{\hbar}: E \rightarrow D$ the quantization maps.

Conventions 2.7. Let $E$ and $D$ be a real vector bundle and a $C^{*}$-algebra bundle over $M$ respectively. Every $\mathbb{R}$-linear bundle map $\varphi: E \rightarrow D$ extends to a $\mathbb{C}$-linear bundle map from the complexified bundle $E \otimes \mathbb{C}$ to $D$ sending $f+i g$ to $\varphi(f)+i \varphi(g)$, where $f, g \in \Gamma(E)$. We'll denote this extended map still by $\varphi$.

Since $\mathfrak{A}_{2 n}$ is unital the bundle $D$ contains the trivial bundle $M \times \mathbb{C}$ as a subbundle naturally. Thus $\Gamma_{\infty}(D)$ contains $C_{\infty}(M)$ as a subalgebra. We are ready to construct strict quantizations for $M$ :

Theorem 2.8. Let $(M, \Pi)$ be a symplectic manifold. Let $J$ be a compatible almost complex structure on $T^{*} M$, and let $D$ and $\varphi_{\hbar}$ be the quantization bundle and maps of $\left(T^{*} M, \Pi, J\right)$. Let $\mathcal{A}_{\hbar}=\Gamma_{\infty}(D)$ and $\pi_{\hbar}(f)=f+\hbar^{\frac{1}{2}} \varphi_{\hbar}(d f)$ for all $0<\hbar \leq 1$ and $f \in C_{c}^{\infty}(M)$. Also let $\mathcal{A}_{0}=C_{\infty}(M)$, and let $\pi_{0}$ be the canonical embedding of $C_{c}^{\infty}(M)$ into $C_{\infty}(M)$. Let $\left\{\mathcal{A}_{\hbar}\right\}$ be the subfield of the trivial continuous field of $C^{*}$-algebras $[0,1] \times \Gamma_{\infty}(D)$ over $[0,1]$. Then $\left\{\mathcal{A}_{\hbar}, \pi_{\hbar}\right\}$ is a faithful flabby strict quantization of $M$ over $I=[0,1]$ with $A=C_{c}^{\infty}(M)$. If a Lie group $G$ has an action on $M$ preserving $\Pi$ and $J$, then this strict quantization is $G$-equivariant.

Proof. We verify the conditions in Definition 1.1 Condition (1) follows from our choice of $\left(\mathcal{A}_{0}, \pi_{0}\right)$. Condition (2) follows from Definition 2.1 (2)(3). Condition (3) follows from Definition 2.1 (1) and our discussion at the beginning of this section. Thus $\left\{A_{\hbar}, \pi_{\hbar}\right\}$ is a strict quantization of $M$. Since $A=C_{c}^{\infty}(M)$ this strict quantization is flabby. The faithfulness follows from Lemma 2.9] below. In fact we need only $\varphi_{\hbar}\left(\mathbb{R}^{2 n} \otimes \mathbb{C}\right) \cap \mathbb{C} 1_{\mathfrak{A}_{2 n}}=\{0\}$ here, but we shall need the full result of Lemma 2.9 later in Corollary [5.6.

Lemma 2.9. Let $\mathfrak{A}_{2 n}$ be a Heisenberg algebra. Let $V_{\hbar}=\varphi_{\hbar}\left(\mathbb{R}^{2 n} \otimes \mathbb{C}\right)+\left(\varphi_{\hbar}\left(\mathbb{R}^{2 n} \otimes\right.\right.$ $\mathbb{C}))^{*}$. Then $V_{\hbar} \cap \mathbb{C}_{\mathfrak{A}_{2 n}}=\{0\}$ for every $0<\hbar \leq 1$. 
Proof. It suffices to show that the only $U(n)$-fixed element in $V_{\hbar}$ is 0 . Endow $U(n)$ with the normalized Haar measure. Let $\sigma$ be the canonical map $\mathfrak{A}_{2 n} \rightarrow\left(\mathfrak{A}_{2 n}\right)^{U(n)}$ defined by $\sigma(a)=\int_{U(n)} \rho_{h}(a) d h$. Similarly define $\tau: \mathbb{R}^{2 n} \otimes \mathbb{C} \rightarrow\left(\mathbb{R}^{2 n} \otimes \mathbb{C}\right)^{U(n)}$. Then $\varphi_{\hbar} \circ \tau=\sigma \circ \varphi_{\hbar}$. Clearly $\left(\mathbb{R}^{2 n}\right)^{U(n)}=\{0\}$, and hence $\tau\left(\mathbb{R}^{2 n} \otimes \mathbb{C}\right)=\{0\}$. Consequently $\left(V_{\hbar}\right)^{U(n)}=\sigma\left(V_{\hbar}\right)=\{0\}$.

Finally, the assertions about $G$-action is clear.

The proof of Theorem 2.8 generalizes to Theorem 2.10 immediately:

Theorem 2.10. Let $(M, \Pi)$ be an almost Poisson manifold. Let $\left(E, \Pi_{E}, J_{E}\right)$ be as in Lemma 2.5. Suppose that $\psi: T^{*} M \rightarrow E$ is an almost Poisson map (Definition [2.4). Let $D$ and $\varphi_{\hbar}$ be the quantization bundle and maps of $\left(E, \Pi_{E}, J_{E}\right)$. Let $\mathcal{A}_{\hbar}=\Gamma_{\infty}(D)$ and $\pi_{\hbar}(f)=f+\hbar^{\frac{1}{2}}\left(\varphi_{\hbar} \circ \psi\right)(d f)$ for all $0<\hbar \leq 1$ and $f \in C_{c}^{\infty}(M)$. Also let $\mathcal{A}_{0}$ and $\varphi_{0}$ be as in Theorem [2.8, Let $\left\{A_{\hbar}\right\}$ be the subfield of the trivial continuous field of $C^{*}$-algebras $[0,1] \times \Gamma_{\infty}(D)$ over $[0,1]$. Then $\left\{A_{\hbar}, \pi_{\hbar}\right\}$ is a faithful flabby strict quantization of $M$ over $I=[0,1]$ with $A=C_{c}^{\infty}(M)$. If a Lie group $G$ has an action on $M$ preserving $\Pi$ and has an action on $\left(E, \Pi_{E}, J_{E}\right)$ such that the projection $E \rightarrow M$ and the map $\psi$ are $G$-equivariant, then this strict quantization is $G$-equivariant.

\section{Strict Quantizations of Almost Poisson Manifolds}

Throughout the rest of this paper $(M, \Pi)$ will be an almost Poisson manifold unless stated otherwise. To construct strict quantizations of $M$, by Theorem 2.10 it suffices to find $\left(E, \Pi_{E}\right)$ and $\psi: T^{*} M \rightarrow E$ as in Theorem 2.10 In fact there is a canonical choice of such $\left(E, \Pi_{E}\right)$ and $\psi$. This follows from Lemmas 3.1 and 3.2 for which we omit the proof.

Lemma 3.1. Let $E \rightarrow M$ be a vector bundle, and let $E^{*}$ be its dual bundle. Then $\Omega_{x}\left(\left(u_{1}, v_{1}^{*}\right),\left(u_{2}, v_{2}^{*}\right)\right)=<u_{1}, v_{2}^{*}>-<u_{2}, v_{1}^{*}>$ defines an almost symplectic structure on $E \oplus E^{*}$, where $u_{j} \in E_{x}, v_{j}^{*} \in E_{x}^{*}$ and $<\cdot, \cdot>$ is the canonical pairing between $E$ and $E^{*}$.

Lemma 3.2. Let $\left(E, \Pi_{E}\right)$ be an almost Poisson bundle over $M$, and let $\sigma: E \rightarrow E^{*}$ be the induced bundle map defined by $\sigma_{x}(u)=\Pi_{E, x}(\cdot, u)$. Let $E \oplus E^{*}$ be endowed with the canonical almost symplectic structure defined in Lemma 3.1. Then the bundle map $\psi: E \rightarrow E \oplus E^{*}$ defined by $\psi_{x}(u)=\left(\frac{1}{\sqrt{2}} u, \frac{1}{\sqrt{2}} \sigma_{x}(u)\right)$ is an almost Poisson map.

By Theorem 2.10 we have:

Theorem 3.3. Let $\Omega_{M}$ be the canonical almost symplectic structure on $T^{*} M \oplus T M$ defined in Lemma 3.1 for $E=T^{*} M$. Let $\psi: T^{*} M \rightarrow T^{*} M \oplus T M$ be the bundle map defined in Lemma 3.2 for $\left(T^{*} M, \Pi\right)$. Let $J$ be a compatible almost complex structure for $\left(T^{*} M \oplus T M, \Omega_{M}\right)$, and let $D$ and $\varphi_{\hbar}$ be the quantization bundle and maps of $\left(T^{*} M \oplus T M, \Omega_{M}, J\right)$. Let $\left\{\mathcal{A}_{\hbar}, \pi_{\hbar}\right\}$ be as in Theorem 2.10. Then $\left\{A_{\hbar}, \pi_{\hbar}\right\}$ is a faithful flabby strict quantization of $M$ over $I=[0,1]$ with $A=C_{c}^{\infty}(M)$. If a Lie group $G$ has a smooth action on $M$ preserving $\Pi$ and $J$, then this strict quantization is G-equivariant.

We are ready to prove Theorem 1.2 
Proof of Theorem 1.2 Given any Riemannian metric on $M$, the induced isomorphism $T M \rightarrow T^{*} M$ induces an inner product on $T^{*} M$, and hence induces an inner product on $T^{*} M \oplus T M$ by requiring $T^{*} M$ and $T M$ to be perpendicular to each other. Therefore $T^{*} M \oplus T M$ has a $G$-invariant inner product. Notice that given an inner product on an almost symplectic bundle $(E, \Omega)$ there is a canonical way to construct a compatible almost complex structure $J$ on $E$ [5]. Thus $T^{*} M \oplus T M$ has a $G$-invariant compatible almost complex structure. Now the assertions follow from Theorem 3.3

Corollary 3.4. For any smooth action of a compact Lie group $G$ on $M$ preserving $\Pi$, there is a $G$-equivariant faithful strict quantization of $M$ over $I=[0,1]$ with $A=C_{c}^{\infty}(M)$.

Proof. For any smooth action of a compact Lie group, the manifold admits an invariant Riemannian metric by integrating any given Riemannian metric.

Rieffel showed 23] that there is no strict deformation quantization of the rotationally invariant symplectic structure on $S^{2}$ respecting the action of $S O(3)$. So this gives us some sign on how more restrictive strict deformation quantizations are than strict quantizations.

Corollary 3.5. If a Lie group $G$ has a bi-invariant Riemannian metric, then $\mathfrak{g}^{*}$ equipped with the Lie-Poisson bracket [6] admits a faithful strict quantization with $A=C_{c}^{\infty}\left(\mathfrak{g}^{*}\right)$ equivariant under the coadjoint action of $G$, where $\mathfrak{g}$ is the Lie algebra of $G$ and $\mathfrak{g}^{*}$ is the dual.

Proof. Identify the cotangent space of $\mathfrak{g}^{*}$ at each point with $\mathfrak{g}$. Then for any $\xi \in \mathfrak{g}^{*}$ and $g \in G$, the isomorphism $T_{\xi}^{*} \mathfrak{g}^{*} \rightarrow T_{A d_{g}^{*}(\xi)}^{*} \mathfrak{g}^{*}$ is exactly $A d_{g}: \mathfrak{g} \rightarrow \mathfrak{g}$. In particular considering $\xi=0$, we see that $\mathfrak{g}^{*}$ admits an invariant Riemannian metric if and only if the vector space $\mathfrak{g}$ has an inner product invariant under the adjoint action of $G$, if and only if $G$ has a bi-invariant Riemannian metric.

Example 3.6. Assume that $T M$ is trivial. Let $X_{1}, \cdots, X_{m} \in \Gamma(T M)$ giving the trivialization of $T M$. Let $X_{1}^{*}, \cdots, X_{m}^{*} \in \Gamma\left(T^{*} M\right)$ be the dual basis. Define $J$ on $T^{*} M \oplus T M$ by $J\left(X_{k}^{*}\right)=X_{k}$ and $J\left(X_{k}\right)=-X_{k}^{*}$ for $1 \leq k \leq m$. Then the quantization bundle $D$ is the trivial bundle $M \times \mathfrak{A}_{2 m}$, and thus $\Gamma_{\infty}(D)=$ $C_{\infty}\left(M, \mathfrak{A}_{2 m}\right)=C_{\infty}(M) \otimes \mathfrak{A}_{2 m}$. Let

$$
\beta_{j k}=\Pi\left(X_{j}^{*}, X_{k}^{*}\right)
$$

be the structure functions. Then the bundle map $\sigma: T^{*} M \rightarrow T M$ is determined by $\sigma\left(X_{k}^{*}\right)=\sum_{j=1}^{m} \beta_{j k} X_{j}$. Thus for any $f, g \in C_{c}^{\infty}(M)$ we have

$$
\begin{aligned}
\{f, g\} & =\sum_{1 \leq j, k \leq m} \beta_{j k} X_{j}(f) X_{k}(g), \\
\pi_{\hbar}(f) & =f \otimes 1+\frac{1}{\sqrt{2}} \hbar^{\frac{1}{2}}\left(\sum_{1 \leq j \leq m} X_{j}(f) \otimes \varphi_{\hbar}\left(e_{j}\right)+\sum_{1 \leq j, k \leq m} \beta_{j k} X_{k}(f) \otimes \varphi_{\hbar}\left(e_{m+j}\right)\right),
\end{aligned}
$$

where $e_{j}$ and $\varphi_{\hbar}$ are as in Definition 2.1

Example 3.7. Let $M$ be the $m$-torus $\mathbb{T}^{m}$, and let $x_{1}, \cdots, x_{m}$ be the standard coordinates. Let $\theta$ be a real skew-symmetric $m \times m$ matrix. Define a Poisson 
bracket $\{\cdot, \cdot\}$ on $M$ by

$$
\{f, g\}=\frac{1}{2} \sum_{1 \leq j, k \leq m} \theta_{j k} \frac{\partial f}{\partial x_{j}} \frac{\partial g}{\partial x_{k}} .
$$

Let $X_{j}=\frac{\partial}{\partial x_{j}}$. Let $\beta_{j k}$ and $J$ be as in Example 3.6 Then $\beta_{j k}=\frac{1}{2} \theta_{j k}$. Thus

$\pi_{\hbar}(f)=f \otimes 1+\frac{1}{\sqrt{2}} \hbar^{\frac{1}{2}}\left(\sum_{1 \leq j \leq m} \frac{\partial f}{\partial x_{j}} \otimes \varphi_{\hbar}\left(e_{j}\right)+\frac{1}{2} \sum_{1 \leq j, k \leq m} \theta_{j k} \frac{\partial f}{\partial x_{k}} \otimes \varphi_{\hbar}\left(e_{m+j}\right)\right)$.

This is very different from Rieffel's Moyal product approach [23] 26], which leads to the noncommutative torus $A_{\theta}$.

Example 3.8. Let $\mathfrak{g}$ be a Lie algebra, and let $M$ be the dual $\mathfrak{g}^{*}$ equipped with the Lie-Poisson bracket. Let $v_{1}, \cdots, v_{m}$ be a basis of $\mathfrak{g}$, and let $\mu_{1}, \cdots, \mu_{m}$ be the dual basis of $\mathfrak{g}^{*}$. Let $c_{j k l}$ be the structure constants satisfying $\left[v_{j}, v_{k}\right]=\sum c_{j k l} v_{l}$. We may take $X_{j}$ in Example 3.6 to be $\mu_{j}$. Then $X_{j}^{*}=v_{j}$, and $\beta_{j k}=\sum_{l} c_{j k l} v_{l}$. Thus

$\pi_{\hbar}(f)=f \otimes 1+\frac{1}{\sqrt{2}} \hbar^{\frac{1}{2}}\left(\sum_{1 \leq j \leq m} \mu_{j}(f) \otimes \varphi_{\hbar}\left(e_{j}\right)+\sum_{1 \leq j, k, l \leq m} c_{j k l} v_{l} \mu_{k}(f) \otimes \varphi_{\hbar}\left(e_{m+j}\right)\right)$.

\section{4. Номотору}

Our construction in Theorem 3.3 depends on the choice of $J$. We define homotopy of strict quantizations first, then show that the homotopy class of our construction in independent of the choice of $J$ (Proposition 4.3). The definition of homotopy of strict quantizations is similar to the usual definition of homotopy of homomorphisms between $C^{*}$-algebras:

Definition 4.1. Let $\left\{\mathcal{A}_{\hbar}^{j}, \pi_{\hbar}^{j}\right\}$ be strict quantizations of $(M, \Pi)$ over $I$ for $A$, where $j=0,1$. By a homotopy of these two strict quantizations, we mean a continuous field of $C^{*}$-algebras $\left\{\mathcal{A}_{\hbar, t}\right\}$ over $I \times[0,1]$ and linear maps $\pi_{\hbar, t}: A \rightarrow \mathcal{A}_{\hbar, t}$ such that

(1) the restriction of this field on $I \times\{t\}$ gives a strict quantization of $(M, \Pi)$ over $A$ for each $t \in[0,1]$ in a uniform way, i.e. for all $f, g \in A$ we have

$$
\lim _{\hbar \rightarrow 0} \sup _{0 \leq t \leq 1}\left\|\left[\pi_{\hbar, t}(f), \pi_{\hbar, t}(g)\right] /(i \hbar)-\pi_{\hbar, t}(\{f, g\})\right\|=0,
$$

(2) for $t=0,1$ the restriction of this field gives the strict quantizations $\left\{\mathcal{A}_{\hbar}^{0}, \pi_{\hbar}^{0}\right\}$ and $\left\{\mathcal{A}_{\hbar}^{1}, \pi_{\hbar}^{1}\right\}$ respectively.

Remark 4.2. One may also define a weaker notion of homotopy without requiring the convergence in (1) to be uniformly. We adopt the stronger one because the homotopies we construct here all satisfy the uniform condition.

Clearly homotopy is an equivalence relation between strict quantizations of $M$.

Proposition 4.3. The homotopy class of the strict quantization in Theorem 3.3 does not depend on the choice of the compatible almost complex structure $J$ on $T^{*} M \oplus T M$.

Proof. Let $J_{0}$ and $J_{1}$ be two compatible almost complex structures on $T^{M} \oplus T M$. Let $<\cdot, \cdot>_{0}$ and $\left.<\cdot, \cdot\right\rangle_{1}$ be the induced inner products. Let $\left.<\cdot, \cdot\right\rangle_{t}=t<$ $\cdot, \cdot>_{1}+(1-t)<\cdot, \cdot>_{0}$ for $0 \leq t \leq 1$. The canonical way of constructing compatible almost complex structure from given inner product [5] is continuous. Thus we get a continuous family of compatible almost complex structures $J_{t}$ on 
$T^{*} M \oplus T M$. Let $\left(D_{t}, \varphi_{\hbar, t}\right)$ be the corresponding quantization bundle and maps. Denote $\Gamma_{c}\left(T^{*} M \oplus T M\right)$ the space of compactly supported sections of $T^{M} \oplus T M$. Then the sections $\left(\varphi_{\hbar, t}(f)\right)_{0 \leq t \leq 1}$ for $0<\hbar \leq 1$ and $f \in \Gamma_{c}\left(T^{*} M \oplus T M\right)$ generate a continuous field of $C^{*}$-algebras over $[0,1]$ with fibre $\Gamma_{\infty}\left(D_{t}\right)$ at $t$. Now it is easy to see that the strict quantizations in Theorem 3.3 associated with $J_{t}$ 's combine together to give a homotopy of the ones associated with $J_{0}$ and $J_{1}$.

For given $(M, \Pi, A, I)$ we don't know whether there is only one homotopy class of strict quantizations. But this is the case when $\Pi=0$ and $I$ is an interval. Notice first that for $\Pi=0$ there is a canonical trivial strict quantization over any $I$, namely $\mathcal{A}_{\hbar}=C_{\infty}(M)$ and $\pi_{\hbar}$ is the canonical inclusion of $A$ into $C_{\infty}(M)$.

Proposition 4.4. When $\Pi=0$ and $I$ is an interval, every strict quantization over $I$ is homotopic to the canonical one.

Proof. Let $\left\{\mathcal{A}_{\hbar}, \varphi_{\hbar}\right\}$ be a strict quantization. Define a map $\gamma: I \times[0,1] \rightarrow I$ by $\gamma(\hbar, t)=t \hbar$. Then the pull back of the field $\left\{\mathcal{A}_{\hbar}\right\}$ under $\gamma$ is a continuous field over $I \times[0,1]$ with fibre $\mathcal{A}_{\hbar, t}=\mathcal{A}_{t \hbar}$ at $(\hbar, t)$. For each $f \in A$ let $\left\{\pi_{\hbar, t}(f)\right\}$ be the pull back of the section $\left\{\pi_{\hbar}(f)\right\}$ under $\gamma$, namely $\pi_{\hbar, t}(f)=\pi_{t \hbar}(f)$. Then clearly this is a homotopy between the canonical strict quantization and $\left\{\mathcal{A}_{\hbar}, \varphi_{\hbar}\right\}$.

\section{Local Strict Quantizations}

There are two different meanings for a strict quantization to be local. The first one is an intuitive one, meaning that the construction is local in the sense that we construct strict quantizations for open subsets of $M$ first, then gluing them together to get strict quantization for $M$. This includes our construction in Theorem 2.10 and the construction in 19. The second one means that the algebras and maps $\left\{\mathcal{A}_{\hbar}, \pi_{\hbar}\right\}$ are local in the sense that $\mathcal{A}_{\hbar} \subseteq \mathcal{D}_{\hbar}$ for some (upper-semi)continuous field of $C^{*}$-algebras $D_{\hbar}$ and the maps $\pi_{\hbar}: C_{\infty}(M)=\Gamma_{\infty}(M \times \mathbb{C}) \rightarrow \mathcal{A}_{\hbar} \hookrightarrow \Gamma_{\infty}\left(D_{\hbar}\right)$ are fibrewise. Here we'll concentrate on the second meaning.

Let $X$ be a locally compact Hausdorff space. Recall that an $C_{\infty}(X)$-algebra is a $C^{*}$-algebra $\mathcal{A}$ with an injective nondegenerate homomorphism $\gamma: C_{\infty}(X) \rightarrow \mathcal{M}(\mathcal{A})$ such that $\gamma\left(C_{\infty}(X)\right)$ being contained in the center $\mathcal{Z M}(\mathcal{A})$ of the multiplier algebra $\mathcal{M}(\mathcal{A})$ [10]. This is equivalent to saying that $\mathcal{A}$ is the global section algebra of an upper-semicontinuous field of $C^{*}$-algebras over $X$ 21]. Under this correspondence the fibre algebra of the field at $x \in X$ is $\mathcal{A} / \overline{\gamma\left(I_{x}\right) \mathcal{A}}$, where $I_{x}=\left\{h \in C_{\infty}(X)\right.$ : $h(x)=0\}$. This motivates our definition of local strict quantizations:

Definition 5.1. Let $\left\{\mathcal{A}_{\hbar}, \pi_{\hbar}\right\}$ be a strict quantization of $(M, \Pi)$ on $I$. We call $\left\{\mathcal{A}_{\hbar}, \pi_{\hbar}\right\}$ local if each $\mathcal{A}_{\hbar}$ is a $C_{\infty}(M)$-algebra with $\gamma_{\hbar}: C_{\infty}(M) \hookrightarrow \mathcal{Z} \mathcal{M}\left(\mathcal{A}_{\hbar}\right)$ such that $\left\|\pi_{\hbar}(f)-\gamma_{\hbar}(f)\right\| \rightarrow 0$ as $\hbar \rightarrow 0$ for every $f \in A$.

Clearly the strict quantizations in Theorem 2.10 are local. But the ones in 19] are not.

Proposition 5.2. Let $\left\{\mathcal{A}_{\hbar}, \pi_{\hbar}\right\}$ be a local strict quantization of $(M, \Pi)$ on I. Let $f, g \in A_{s a}$. If $\pi_{\hbar}(f), \pi_{\hbar}(g) \in\left(\mathcal{A}_{\hbar}\right)_{s a}$ for all $\hbar \in I$, then $\{f, g\}=0$.

Proof. Using the embeddings $\gamma_{\hbar}$ in Definition 5.1 we'll identify $C_{\infty}(M)$ as a subalgebra of $\mathcal{M}\left(\mathcal{A}_{\hbar}\right)$. Then $\left\|\pi_{\hbar}(\{f, g\})-\{f, g\}\right\| \rightarrow 0$ as $\hbar \rightarrow 0$. Thus Definition 1.13) becomes

$$
\lim _{\hbar \rightarrow 0}\left\|\left[\pi_{\hbar}(f), \pi_{\hbar}(g)\right] /(i \hbar)-\{f, g\}\right\|=0 .
$$


For $x \in M$ let $I_{x}=\left\{h \in C_{\infty}(M): h(x)=0\right\}$, and let $\mathcal{A}_{\hbar, x}=\mathcal{A}_{\hbar} / \overline{I_{x} \mathcal{A}_{\hbar}}$. Let $\beta_{\hbar, x}: \mathcal{A}_{\hbar} \rightarrow \mathcal{A}_{\hbar, x}$ be the quotient map. Notice that the identity of $\mathcal{M}\left(\mathcal{A}_{\hbar, x}\right)$ is $\beta_{\hbar, x}(h)$ for any $h \in C_{\infty}(M)$ with $h(x)=1$. Taking $\beta_{\hbar, x}$ on (4) we get

$$
\lim _{\hbar \rightarrow 0}\left\|\left[\beta_{\hbar, x}\left(\pi_{\hbar}(f)\right), \beta_{\hbar, x}\left(\pi_{\hbar}(g)\right)\right] /(i \hbar)-\{f, g\}(x)\right\|=0 .
$$

Notice that $\left[\beta_{\hbar, x}\left(\pi_{\hbar}(f)\right), \beta_{\hbar, x}\left(\pi_{\hbar}(g)\right)\right] /(i \hbar)$ is a self-commutator, i.e. of the form $\left[S^{*}, S\right]$ for some $S$ (for instance $S=(2 \hbar)^{-1 / 2} \beta_{\hbar, x}\left(\pi_{\hbar}(f)\right)-i(2 \hbar)^{-1 / 2} \beta_{\hbar, x}\left(\pi_{\hbar}(g)\right)$ ). It is known that self-commutators can't be invertible [22 Corollary 1]. Thus $\{f, g\}(x)=0$.

Corollary 5.3. An almost Poisson manifold $(M, \Pi)$ admits a Hermitian local strict quantization if and only if $\Pi=0$.

Proof. Assume that $\Pi \neq 0$ and that $M$ admits a Hermitian local strict quantization. Then we can find a covector field $Y^{*} \in \Gamma\left(T^{*} M\right)$ with $\sigma\left(Y^{*}\right) \neq 0$, where $\sigma$ is as in Lemma 3.2 for $E=T^{*} M$. We claim that there is a vector field $X \neq 0$ such that $X(d f)=0$ for all $f \in A$. If $\sigma(d g) \neq 0$ for some $g \in A_{s a}$, by Proposition 5.2 we may take $X=\sigma(d g)$. Otherwise we may take $X=\sigma\left(Y^{*}\right)$. Let $Z$ be a nonconstant integral curve of $X$. Then the restriction of every $f \in A$ on $Z$ is constant, which contradicts $A$ being dense in $C_{\infty}(M)$. This proves the "only if" part. The "if" part is trivial.

Remark 5.4. We don't know when a local strict quantization is homotopic to a Hermitian strict quantization. As a comparison, a star product on a symplectic manifold is equivalent to a Hermitian one if and only if its characteristic class is Hermitian [20. Also every Poisson manifold has Hermitian star products 4].

Thus the strict quantizations in Theorem 2.10 are not Hermitian unless $\Pi=0$. In fact we can say more:

Proposition 5.5. Let $\left\{\mathcal{A}_{\hbar}, \pi_{\hbar}\right\}$ be a local strict quantization of $(M, \Pi)$ on I. Identify $C_{\infty}(M)$ as a subalgebra of $\mathcal{M}\left(\mathcal{A}_{\hbar}\right)$ via $\gamma_{\hbar}$. Let $\tau_{\hbar}(f)=\pi_{\hbar}(f)-f$ for $0<\hbar \leq 1$ and $f \in A$. Assume that

$$
A \cap\left(\tau_{\hbar}(A)+\left(\tau_{\hbar}(A)\right)^{*}\right)=\{0\}
$$

for every $0<\hbar \leq 1$. If this is a strict deformation quantization, then it is Hermitian and $\Pi=0$.

Proof. Let $f \in A$ and $0<\hbar \leq 1$. Then $\left(\pi_{\hbar}(f)\right)^{*}=\pi_{\hbar}(g)$ for some $g \in A$. Thus $f^{*}+\left(\tau_{\hbar}(f)\right)^{*}=g+\tau_{\hbar}(g)$. By our assumption $f^{*}=g$. Thus this strict quantization is Hermitian. By Corollary $5.3 \Pi=0$.

Corollary 5.6. Let $\left\{\mathcal{A}_{\hbar}, \pi_{\hbar}\right\}$ be a local strict quantization of $(M, \Pi)$ on $I$ with $\Pi \neq 0$. Let $\tau_{\hbar}$ be as in Proposition [5.5 and assume that (6) holds for all $0<$ $\hbar \leq 1$. Then $\left\{\mathcal{A}_{\hbar}, \pi_{\hbar}\right\}$ can't be restricted to a dense $*$-subalgebra of $A$ to get a strict deformation quantization of $(M, \Pi)$. In particular, the strict quantizations in Theorem 2.10 can't be restricted to a dense $*$-subalgebra of $C_{c}^{\infty}(M)$ to get a strict deformation quantization unless $\Pi=0$.

Proof. By Lemma 2.9 the strict quantizations in Theorem 2.10) satisfy (6).

Corollary 5.7. For any $(M, \Pi)$ there is a faithful flabby strict quantization, which can't be restricted to any dense *-subalgebra of $A$ to get a strict deformation quantization. 
Proof. The case $\Pi \neq 0$ follows from Theorem 3.3 and Corollary 5.6. The case $\Pi=0$ is settled in [16].

Corollary [5.7 gives Question 25 in [27] a negative answer, which asks whether there is an example of a faithful strict quantization of a Poisson manifold such that it's impossible to restrict it to some dense $*$-subalgebra to get a strict deformation quantization. This leaves the question whether we can require the strict quantization to be Hermitian.

\section{Functorial Properties}

It is unlikely that there is a universal way to construct a canonical strict quantization for each Poisson manifolds such that it gives a contravariant functor from the category of Poisson manifolds with (proper) Poisson maps to the category of continuous field of $C^{*}$-algebras over $I$ [14]. Instead, Landsman proposed other categories closely related to Morita equivalence, and showed that there is such a functor on the subcategory of dual of integrable Lie algebroids.

Though our construction in Theorem 3.3 doesn't give a contravariant functor, it does has some properties similar to functors. In this section we discuss two questions:

(1) fixing a strict quantization $\left\{\mathcal{A}_{\hbar}^{M}, \pi_{\hbar}^{M}\right\}$ of $\left(M, \Pi_{M}\right)$ on $I$, for any proper Poisson map $\phi$ from $M$ to another almost Poisson manifold $\left(N, \Pi_{N}\right)$ can we find a strict quantization $\left\{\mathcal{A}_{\hbar}^{N}, \pi_{\hbar}^{N}\right\}$ of $\left(N, \Pi_{N}\right)$ on $I$ with a "homomorphism" of these two strict quantizations extending $\phi^{*}: C_{\infty}(N) \rightarrow C_{\infty}(M)$, i.e. a homomorphism $\xi_{\hbar}: \mathcal{A}_{\hbar}^{N} \rightarrow \mathcal{A}_{\hbar}^{M}$ for each $\hbar \in I$ such that these maps $\left\{\phi_{\hbar}\right\}$ send continuous sections to continuous ones and $\xi_{\hbar} \circ \pi_{\hbar}^{N}=\pi_{\hbar}^{M} \circ \phi^{*}$ ?

(2) the similar question but fixing the strict quantization of $N$ instead.

The first question has a positive answer because of Theorem 1.2 and the following Proposition, whose proof is just routine verification.

Proposition 6.1. Let $\left\{\mathcal{A}_{\hbar}^{M}, \pi_{\hbar}^{M}\right\}$ and $\left\{\mathcal{A}_{\hbar}^{N}, \pi_{\hbar}^{N}\right\}$ be strict quantizations of $\left(M, \Pi_{M}\right)$ and $\left(N, \Pi_{N}\right)$ on $I$ for $A^{M}$ and $A^{N}$ respectively. Let $\phi: M \rightarrow N$ be a proper Poisson map with $\phi^{*}\left(A^{N}\right) \subseteq A^{M}$. Then the sections $\left\{(f, g): f \in \Gamma\left(\left\{\mathcal{A}_{\hbar}^{M}\right\}\right), g \in\right.$ $\left.\Gamma\left(\left\{\mathcal{A}_{\hbar}^{N}\right\}\right), f_{0}=\phi^{*}\left(g_{0}\right)\right\}$ determine a continuous field of $C^{*}$-algebras over I with fibre $\mathcal{A}_{\hbar}^{M} \oplus \mathcal{A}_{\hbar}^{N}$ at $\hbar \neq 0$ and fibre $C_{\infty}(M)$ at $\hbar=0$. And $\left(\pi_{\hbar}^{M} \circ \phi^{*}\right) \oplus \pi_{\hbar}^{N}$ for $\hbar \neq 0$ give a strict quantization for $\left(N, \Pi_{N}\right)$ on I for $A^{N}$.

Remark 6.2. (1) When both $\left\{\mathcal{A}_{\hbar}^{M}, \pi_{\hbar}^{M}\right\}$ and $\left\{\mathcal{A}_{\hbar}^{N}, \pi_{\hbar}^{N}\right\}$ are local in the sense of Definition 5.1 so is $\left\{\mathcal{A}_{\hbar}^{M} \oplus \mathcal{A}_{\hbar}^{N},\left(\pi_{\hbar}^{M} \circ \phi^{*}\right) \oplus \pi_{\hbar}^{N}\right\}$;

(2) When $\left(M, \Pi_{M}\right)=\left(N, \Pi_{N}\right)$ and $\phi=i d_{M}$, Proposition 6.1] shows that the set of isomorphism classes of strict quantizations of $(M, \Pi)$ over $I$ for $A$ has a natural abelian semigroup structure. Clearly the addition is compatible with homotopy defined in Definition 4.1] Thus the set of homotopy classes of strict quantizations of $(M, \Pi)$ over $I$ for $A$ is also an abelian semigroup.

For the second question we have a partial positive answer:

Proposition 6.3. Let $\left(N, \Pi_{N}\right)$ be an almost symplectic manifold, and let $k \geq$ $\operatorname{dim} N$. Then there is a strict quantization $\left\{\mathcal{A}_{\hbar}^{N}, \pi_{\hbar}^{N}\right\}$ of $\left(N, \Pi_{N}\right)$ as constructed in Theorem 3.3 such that for any proper Poisson map $\phi:\left(M, \Pi_{M}\right) \rightarrow\left(N, \Pi_{N}\right)$ with $k \geq \operatorname{dim} M$ there is a strict quantization $\left\{\mathcal{A}_{\hbar}^{M}, \pi_{\hbar}^{M}\right\}$ of $\left(M, \Pi_{M}\right)$ as constructed in 
Theorem 3.3 and homomorphisms $\xi_{\hbar}: \mathcal{A}_{\hbar}^{N} \rightarrow \mathcal{A}_{\hbar}^{M}$ sending continuous sections to continuous ones with $\xi_{\hbar} \circ \pi_{\hbar}^{N}=\pi_{\hbar}^{M} \circ \phi^{*}$.

Proof. Let $n=\operatorname{dim} N$, and let $n \leq m \leq k$. We'll choose a special asymptotic representation of Heisenberg commutation relations of dimension $2 m$. Let $e_{1}, \ldots, e_{2 m}$ and $e_{1}^{\prime}, \cdots, e_{2 k}^{\prime}$ be the standard basis of $\mathbb{R}^{2 m}$ and $\mathbb{R}^{2 k}$ respectively. Then the linear map $\eta: \mathbb{R}^{2 m} \rightarrow \mathbb{R}^{2 k}$ defined by $\eta\left(e_{j}\right)=e_{j}^{\prime}, \eta\left(e_{j+m}\right)=e_{j+k}^{\prime}$ for $1 \leq j \leq m$ preserves the standard symplectic structure and the standard compatible almost complex structure. Thus $U(m)$ can be thought of as the subgroup of $U(k)$ fixing $e_{l}^{\prime}, e_{l+k}^{\prime}$ for $m<l \leq k$. Let $\left(\mathfrak{A}_{2 k}, \varphi_{\hbar}\right)$ be an asymptotic representation of Heisenberg commutation relations of dimension $2 k$. Let $\mathfrak{A}_{2 m}$ be the $C^{*}$-subalgebra generated by $\cup_{0<\hbar \leq 1}\left(\varphi_{\hbar} \circ \eta\right)\left(\mathbb{R}^{2 m}\right)$. Then $\left(\mathfrak{A}_{2 m}, \varphi_{\hbar} \circ \eta\right)$ is an asymptotic representation of Heisenberg commutation relations of dimension $2 m$.

Fix a compatible almost complex structure $J^{N}$ on $T^{*} N \oplus T N$. Let $x \in M$. Since $\left(N, \Pi_{N}\right)$ is almost symplectic, $\left(T^{*} \phi\right)_{\phi(x)}$ is injective. Thus $n \leq \operatorname{dim} M$. Let $\left(D^{N}, \sigma^{N}, \varphi_{\hbar}^{N}\right)$ and $\sigma^{M}$ be as in Theorem 3.3 and Lemma 3.2 for $N$ and $M$ respectively. Since $\left(N, \Pi_{N}\right)$ is almost symplectic, $\sigma^{N}$ is invertible. Then we have a linear map $\theta_{x}:=\sigma_{x}^{M} \circ\left(T^{*} \phi\right)_{\phi(x)} \circ\left(\sigma_{\phi(x)}^{N}\right)^{-1}: T N_{\phi(x)} \rightarrow T M_{x}$. Let $\zeta_{x}:=\left(T^{*} \phi\right)_{\phi(x)} \oplus \theta_{x}: T^{*} N_{\phi(x)} \oplus T N_{\phi(x)} \rightarrow T^{*} M_{x} \oplus T M_{x}$. Easy computation shows that $T M_{x} \circ \theta_{x}$ is the identity map on $T N_{\phi(x)}$, and that $\zeta_{x}$ preserves the canonical symplectic structure on $T^{*} N_{x} \oplus T N_{x}$. Then $\left\{\zeta_{x}\left(T^{*} N_{\phi(x)} \oplus T N_{\phi(x)}\right)\right\}$ is an almost symplectic subbundle of $T^{*} M \oplus T M$, which we'll denote by $E$. Let $F_{x}=\left(E_{x}\right)^{\perp}$ with respect to the almost symplectic structure. Then $\left\{F_{x}\right\}$ is also an almost symplectic subbundle of $T^{*} M \oplus T M$, which we'll denote by $F$. Clearly $T^{*} M \oplus T M=E \oplus F$, and $J^{N}$ induces a compatible almost complex structure $J^{E}$ on $E$. Take a compatible almost complex structure $J^{F}$ on $F$. Then $J^{M}:=J^{E} \oplus J^{F}$ is a compatible almost complex structure on $T^{*} M \oplus T M$. Let $\left(D^{M}, \varphi_{\hbar}^{N}\right)$ be as in Theorem 3.3 for $\left(M, \Pi_{M}, J^{M}\right)$.

Say $m=\operatorname{dim} M$. Notice that

$$
\zeta_{x}\left(u_{1}\right), \cdots, \zeta_{x}\left(u_{n}\right), \mu_{1}, \cdots, \mu_{m-n}, \zeta_{x}\left(v_{1}\right), \ldots, \zeta_{x}\left(v_{n}\right), \gamma_{1}, \cdots, \gamma_{m-n}
$$

is a unitary basis of $T^{*} M_{x} \oplus T M_{x}$ for any unitary basis $u_{1}, \cdots, u_{n}, v_{1}, \cdots, v_{n}$ of $T^{*} N_{\phi(x)} \oplus T N_{\phi(x)}$ and any unitary basis $\mu_{1}, \cdots, \mu_{m-n}, \gamma_{1}, \cdots, \gamma_{m-n}$ of $F_{x}$. Because of our choice of $\mathfrak{A}_{2 m}$ and $\mathfrak{A}_{2 n}$ the map $\zeta_{x}$ determines a unital $C^{*}$-algebra embedding $\xi_{x}: D_{\phi(x)}^{N} \rightarrow D_{x}^{M}$ such that $\varphi_{\hbar, x}^{M} \circ \zeta_{x}=\xi_{x} \circ \varphi_{\hbar, \phi(x)}^{N}$ for all $0<\hbar \leq 1$. Since $\phi$ is proper, the $\xi_{x}$ 's combine to give a homomorphism $\xi: \Gamma_{\infty}\left(D^{N}\right) \rightarrow \Gamma_{\infty}\left(D^{M}\right)$ whose restriction on $C_{\infty}(N)$ is $\psi^{*}$. Clearly $\xi_{\hbar}:=\xi$ satisfy the requirement.

Remark 6.4. (1) When both $M$ and $N$ have $G$-equivariant Riemannian metrics there is an obvious $G$-equivariant version of Proposition 6.3

(2) If we can find a $C^{*}$-algebra $\mathfrak{A}_{\infty}$ with an action of $U(\infty):=\cup_{n \in \mathbb{N}} U(n)$ and linear maps $\varphi_{\hbar}^{n}: \mathbb{R}^{2 n} \rightarrow \mathfrak{A}_{\infty}$ for all $n \in \mathbb{N}$ compatible with the embedding $\eta$ : $\mathbb{R}^{2 n} \rightarrow \mathbb{R}^{2 k}$ in the proof of Proposition 6.3 such that for each $n$ these maps give an asymptotic representation of Heisenberg commutation relations, then we can use $\mathfrak{A}_{\infty}$ instead of $\mathfrak{A}_{2 k}$ in the above proof and hence throw away the requirement $k \geq \operatorname{dim} M$. But we don't know whether such infinite dimensional asymptotic representation of Heisenberg commutation relations exists or not. 


\section{Asymptotic Representation of Heisenberg Commutation Relations}

In this section we prove Theorem 2.3

Lemma 7.1. Let $H$ be a separable Hilbert space with orthonormal basis $\left\{e_{j}\right\}_{j=1}^{\infty} \cup$ $\left\{e_{j}^{\prime}\right\}_{j=1}^{\infty}$. Then there exist norm continuous paths $T(\hbar), S(\hbar)$ of operators in $B(H)$ for $0<\hbar \leq 1$ such that

(1) $[T(\hbar), S(\hbar)]\left(e_{2 j-1}\right)=(1+\hbar) i e_{2 j-1}$,

(2) $[T(\hbar), S(\hbar)]\left(e_{2 j}\right)=(1-\hbar) i e_{2 j}$,

(3) $[T(\hbar), S(\hbar)]\left(e_{j}^{\prime}\right)=i e_{j}^{\prime}$,

(4) $\hbar\|T(\hbar)\|$ and $\hbar\|S(\hbar)\|$ are bounded uniformly in $\hbar$.

Brown and Pearcy [3] proved that for a separable Hilbert space $H$ an operator $R \in B(H)$ is a commutator if and only if it is not a non-zero scalar modulo compact operators. For the "if" part, their proof is constructive. Since we need $T(\hbar)$ and $S(\hbar)$ to be continuous, and want some control on their norms, and the construction in [3] depends on some choices of isomorphisms of Hilbert spaces, we write down the proof of Lemma 7.1 here, though it is just following the construction in [3].

Proof. Let $\eta_{j}=\frac{1}{\sqrt{2}}\left(e_{2 j-1}+e_{2 j}\right), \eta_{j}^{\prime}=\frac{1}{\sqrt{2}}\left(e_{2 j-1}-e_{2 j}\right)$. Define $R(\hbar) \in B(H)$ by $R(\hbar)\left(e_{2 j-1}\right)=(1+\hbar) i e_{2 j-1}, R(\hbar)\left(e_{2 j}\right)=(1-\hbar) i e_{2 j}$ and $R(\hbar)\left(e_{j}^{\prime}\right)=i e_{j}^{\prime}$. Define $Z(\hbar) \in B(H)$ by $Z(\hbar)\left(\eta_{j}\right)=\eta_{j}, Z(\hbar)\left(e_{i}^{\prime}\right)=e_{j}^{\prime}$ and $Z(\hbar)\left(\eta_{j}^{\prime}\right)=\eta_{j}+\hbar \eta_{j}^{\prime}$. Then on $\operatorname{span}_{\mathbb{C}}\left\{e_{2 j-1}, e_{2 j}\right\}$, with $\eta_{j}, \eta_{j}^{\prime}$ as basis, $Z(\hbar)$ and $Z(\hbar)^{-1}$ are $\left(\begin{array}{ll}1 & 1 \\ 0 & \hbar\end{array}\right)$ and $\hbar^{-1}\left(\begin{array}{rr}\hbar & -1 \\ 0 & 1\end{array}\right)$ respectively. Therefore $\|Z(\hbar)\|<3$ and $\left\|Z(\hbar)^{-1}\right\|<3 \hbar^{-1}$. Hence it suffices to find continuous paths $T(\hbar), S(\hbar)$ such $[T(\hbar), S(\hbar)]=Z(\hbar)^{-1} R(\hbar) Z(\hbar)$ and $\|T(\hbar)\|,\|S(\hbar)\|$ are bounded uniformly in $\hbar$.

If we identify $H$ with the closure span of $\left\{e_{i}^{\prime}\right\}_{1}^{\infty},\left\{\eta_{i}^{\prime}\right\}_{1}^{\infty},\left\{\eta_{i}\right\}_{1}^{\infty}$ respectively and hence identify $H$ with $H \oplus H \oplus H$, simple calculation shows that

$$
Z(\hbar)^{-1} R(\hbar) Z(\hbar)=i\left(\begin{array}{ccc}
I & 0 & 0 \\
0 & 2 I & I \\
0 & \left(\hbar^{2}-1\right) I & 0
\end{array}\right) .
$$

If we identify $H$ with the closure span of $e_{1}^{\prime}, \eta_{1}^{\prime}, e_{2}^{\prime}, \eta_{2}^{\prime}, \ldots$, and hence identify $H$ with $H \oplus H$, we see that $Z(\hbar)^{-1} R(\hbar) Z(\hbar)$ is of the form $\left(\begin{array}{cc}A & W \\ B & 0\end{array}\right)$, where $A, W$ don't depend on $\hbar$ and $W$ is an isometry with $\operatorname{dim}\left(\operatorname{ker}\left(W^{*}\right)\right)=\infty$ and $B$ is continuous for $0<\hbar \leq 1$ with $\|B\| \leq 1$. By Lemma 5.1 of [3] we can find $X \in B(H)$ such that $A+W X=\left[B_{1}, B_{2}\right]$ for some $B_{1}, B_{2} \in B(H)$ and $X W=0$. Replacing $B_{1}$ by some $B_{1}+\lambda I$ we may assume that $B_{1}-I$ is invertible. By the similarity transformation

$$
\left(\begin{array}{cc}
I & 0 \\
-X & I
\end{array}\right)\left(\begin{array}{ll}
A & W \\
B & 0
\end{array}\right)\left(\begin{array}{ll}
I & 0 \\
X & I
\end{array}\right)=\left(\begin{array}{cc}
A+W X & W \\
-X A-X W X+B & 0
\end{array}\right)
$$

it suffices to find continuous paths $T(\hbar), S(\hbar)$ such that

$$
[T(\hbar), S(\hbar)]=\left(\begin{array}{cc}
A+W X & W \\
-X A-X W X+B & 0
\end{array}\right)
$$

and $\|T(\hbar)\|,\|S(\hbar)\|$ are bounded uniformly in $\hbar$. Simple calculation shows that $T(\hbar)=\left(\begin{array}{cc}B_{1} & 0 \\ 0 & I\end{array}\right), S(\hbar)=\left(\begin{array}{cc}B_{2} & \left(B_{1}-I\right)^{-1} W \\ (-X A-X W X+B)\left(I-B_{1}\right)^{-1} & 0\end{array}\right)$ 
satisfy the requirements.

Lemma 7.2. Let $H$ be a separable Hilbert space. Then for any $n \in \mathbb{N}$ there exist norm continuous paths $T_{1}(\hbar), T_{2}(\hbar), \cdots, T_{n}(\hbar), S_{1}(\hbar), S_{2}(\hbar), \cdots, S_{n}(\hbar)$ of operator in $B(H)$ for $0<\hbar \leq 1$ such that

(1) $\lim _{\hbar \rightarrow 0}\left\|\left[T_{j}(\hbar), S_{k}(\hbar)\right]-\delta_{k}^{j} i\right\|=0$,

(2) $\left[T_{j}(\hbar), T_{k}(\hbar)\right]=\left[S_{j}(\hbar), S_{k}(\hbar)\right]=0$,

(3) $\hbar^{-1 / 3}\left\|T_{j}(\hbar)\right\|$ and $\hbar^{-1 / 3}\left\|S_{j}(\hbar)\right\|$ are bounded uniformly in $\hbar$.

Proof. Let $T(\hbar)$ and $S(\hbar)$ be as in Lemma 7.1 Let

$$
\begin{aligned}
& T_{j}(\hbar)=I \otimes I \otimes \cdots \otimes I \otimes T\left(\hbar^{1 / 3}\right) \otimes I \otimes \cdots \otimes I, \\
& S_{j}(\hbar)=I \otimes I \otimes \cdots \otimes I \otimes S\left(\hbar^{1 / 3}\right) \otimes I \otimes \cdots \otimes I,
\end{aligned}
$$

where $T\left(\hbar^{1 / 3}\right), S\left(\hbar^{1 / 3}\right)$ are at the j-th place. Identify $H$ with $H^{\otimes n}$. Then clearly $T_{j}(\hbar), S_{j}(\hbar)$ satisfy the conditions.

Proof of Theorem [2.3, Let $H$ and $T_{j}(\hbar), S_{j}(\hbar)$ be as in Lemma 7.2 For each $0<$ $\hbar \leq 1$ define a $\mathbb{R}$-linear map $\phi_{\hbar}: \mathbb{R}^{2 n} \rightarrow B(H)$ by $\phi_{\hbar}\left(e_{j}\right)=T_{j}(\hbar), \phi_{\hbar}\left(e_{j+n}\right)=S_{j}(\hbar)$ for $1 \leq j \leq n$, where $e_{1}, \cdots, e_{2 n}$ is the standard basis of $\mathbb{R}^{2 n}$. Clearly $\left\{\phi_{\hbar}\right\}$ satisfy the conditions (1)-(3) in Definition 2.1

Denote the action of $U(n)$ on $\mathbb{R}^{2 n}$ by $\sigma$. Consider the product $C^{*}$-algebra $\prod_{h \in U(n)} B(H)$, whose elements are bounded maps $f: U(n) \rightarrow B(H)$. There is a natural (discontinuous) action $\rho$ of $U(n)$ on $\prod_{h \in U(n)} B(H)$ given by $\rho_{g}(f)(h)=$ $f\left(g^{-1} h\right)$. For each $0<\hbar \leq 1$ define a $\mathbb{R}$-linear map $\varphi_{\hbar}: \mathbb{R}^{2 n} \rightarrow \prod_{h \in U(n)} B(H)$ by $\varphi_{\hbar}(u)(h)=\phi_{\hbar}\left(\sigma_{h^{-1}}(u)\right)$ for $u \in \mathbb{R}^{2 n}$. Clearly $\varphi_{\hbar}$ is $U(n)$-equivariant. Let $\mathfrak{A}_{2 n}$ be the $C^{*}$-subalgebra of $\prod_{h \in U(n)} B(H)$ generated by $\cup_{0<\hbar \leq 1} \varphi_{\hbar}\left(\mathbb{R}^{2 n}\right)$. Then the restriction of $\rho$ on $\mathfrak{A}_{2 n}$ is continuous. Clearly $\left\|\varphi_{\hbar}\right\|=\left\|\phi_{\hbar}\right\|$ and $\left\|\varphi_{\hbar}-\varphi_{\hbar^{\prime}}\right\|=\|$ $\phi_{\hbar}-\phi_{\hbar^{\prime}} \|$, which verify the conditions (2) and (3) of Definition 2.1 Using Lemma $7.2(2)$ simple calculation shows that

$$
\max _{1 \leq j<k \leq 2 n}\left\|\left[\varphi_{\hbar}\left(e_{j}\right), \varphi_{\hbar}\left(e_{k}\right)\right]-\delta_{k-n}^{j} i\right\| \leq \max _{1 \leq j, k \leq n}\left\|\left[T_{j}(\hbar), S_{k}(\hbar)\right]-\delta_{k}^{j} i\right\| .
$$

Then Definition 2.1(1) is also satisfied. In particular, when $\hbar$ is small enough we have $\left\|\left[\varphi_{\hbar}\left(e_{1}\right), \varphi_{\hbar}\left(e_{n+1}\right)\right]-i\right\|<1$. Then $\left[\varphi_{\hbar}\left(e_{1}\right), \varphi_{\hbar}\left(e_{n+1}\right)\right]$ is invertible in $\prod_{h \in U(n)} B(H)$ and hence $\mathfrak{A}_{2 n}$ contain the identity of $\prod_{h \in U(n)} B(H)$.

\section{REFERENCES}

[1] Bayen, F., Flato, M., Frønsdal, C., Lichnerowicz, A., Sternheimer, D.: Deformation theory and quantization, I, II. Ann. Phys. 111.1 (1978), 61-110, 111-151.

[2] Bordemann, M., Meinrenken, E., Schlichenmaier, M.: Toeplitz quantization of Kähler manifolds and $\mathrm{gl}(N), \quad N \rightarrow \infty$ limit. Comm. Math. Phys. 165.2 (1994), 281-296. arXiv:hep-th/9309134

[3] Brown, A., Pearcy, C.: Structure of commutators of operators. Ann. of Math. 82 (1965), $112-127$.

[4] Bursztyn, H., Waldmann, S.: On positive deformations of *-algebras. In: Conférence Moshé Flato 1999, Vol. II (Dijon), Math. Phys. Stud. no. 22, 69-80. Kluwer Acad. Publ., Dordrecht, 2000. math.QA/9910112

[5] Cannas da Silva, A.: Lectures on Symplectic Geometry. Lecture Notes in Mathematics no. 1764. Springer-Verlag, Berlin, 2001.

[6] Cannas da Silva, A., Weinstein, A.: Geometric Models for Noncommutative Algebras. Berkeley Mathematics Lecture Notes no. 10. American Mathematical Society, Providence, RI; Berkeley Center for Pure and Applied Mathematics, Berkeley, CA, 1999. 
[7] De Wilde, M., Lecomte, P. B.: Existence of star-products and of formal deformations of the Poisson Lie algebra of arbitrary sympletic manifolds. Lett. Math. Phys. 7.6 (1983), 487-496.

[8] Dixmier, J.: $C^{*}$-algebras. North-Holland Publishing Co., Amsterdam-New York-Oxford, 1977. Translated from the French by Francis Jellett. North-Holland Mathematical Library, Vol. 15.

[9] Gerstenhaber, M.: On the deformation of rings and algebras. Ann. of Math. 79 (1964), 59-103.

[10] Kasparov, G. G.: Equivariant KK-theory and the Novikov conjecture. Invent. Math. 91.1 (1988), 147-201.

[11] Klimek, S., Lesniewski, A.: Quantum Riemann surfaces for arbitrary Planck's constant. J. Math. Phys. 37.5 (1996), 2157-2165.

[12] Kontsevich, M.: Deformation quantization of Poisson manifolds, I (1997). arXiv:q-alg/9709040

[13] Landsman, N. P.: Mathematical Topics between Classical and Quantum Mechanics. Springer Monographs in Mathematics. Springer-Verlag, New York, 1998.

[14] Landsman, N. P.: Quantization as a functor (2001). arXiv:math-ph/0107023

[15] Landsman, N. P., Ramazan, B.: Quantization of Poisson algebras associated to Lie algebroids. In: Groupoids in analysis, geometry, and physics (Boulder, CO, 1999), Contemp. Math. no. 282, 159-192. Amer. Math. Soc., Providence, RI, 2001. arXiv:math-ph/0001005

[16] LI, H.: Flabby strict deformation quantizations and K-groups (2003). arXiv:math.QA/0303183

[17] Natsume, T.: $C^{*}$-algebraic deformation quantization of closed Riemann surfaces. In: $C^{*}$ algebras (Mnster, 1999), 142-150. Springer, Berlin, 2000.

[18] Natsume, T., Nest, R.: Topological approach to quantum surfaces. Comm. Math. Phys. 202.1 (1999), 65-87.

[19] Natsume, T., Nest, R., Peter, I.: Strict quantizations of symplectic manifolds (2002). preprint.

[20] Neumaier, N.: Local $\nu$-Euler Derivations and Deligne's Characteristic Class of Fedosov Star Products and Star Products of Special Type. Commun. Math. Phys. 230 (2002), 271288. arXiv:math.QA/9905176

[21] NilsEn, M.: $C^{*}$-bundles and $C_{0}(X)$-algebras. Indiana Univ. Math. J. 45.2 (1996), 463-477.

[22] Radjavi, H.: Structure of $A^{*} A-A A^{*}$. J. Math. Mech. 16 (1966), 19-26.

[23] Rieffel, M. A.: Deformation quantization of Heisenberg manifolds. Comm. Math. Phys. 122.4 (1989), 531-562.

[24] Rieffel, M. A.: Deformation quantization and operator algebras. In: Operator theory: operator algebras and applications, Part 1 (Durham, NH, 1988), Proc. Sympos. Pure Math., 51, Part 1, 411-423. Amer. Math. Soc., Providence, RI, 1990.

[25] RiEffel, M. A.: Lie group convolution algebras as deformation quantizations of linear Poisson structures. Amer. J. Math. 112 (1990), 657-686.

[26] Rieffel, M. A.: Deformation Quantization for Actions of $\mathbb{R}^{d}$. Mem. Amer. Math. Soc. no. 506. Amer. Math. Soc., Providence, RI, 1993.

[27] Rieffel, M. A.: Questions on quantization. In: Operator algebras and operator theory (Shanghai, 1997), 315-326. Amer. Math. Soc., Providence, RI, 1998. arXiv:quant-ph/9712009

Department of Mathematics, University of Toronto, Toronto On M5S 3G3, CANADA

E-mail address: hli@fields.toronto.edu 